\title{
Influence of Dynamic Leaks in Volume-Targeted Pressure Support Noninvasive Ventilation: A Bench Study
}

\author{
Manel Luján MD PhD, Ana Sogo MD, Carles Grimau MD, Xavier Pomares MD, \\ Lluís Blanch MD PhD, and Eduard Monsó MD PhD
}

\begin{abstract}
INTRODUCTION: The effect of leaks on volume-targeted pressure support noninvasive ventilation mode has only been tested with continuous simulated leaks. The objective of the study was to assess the influence of random leaks occurring either during inspiration or expiration. METHODS: Analysis of the volume-targeted pressure support mode in 6 commercial ventilators with single-limb circuits and intentional leak in a bench study (restrictive model). Unintentional leaks were introduced through a mechanical system during inspiration (threshold valve with 2 levels of leaks) or during expiration (active valve). Results of delivered tidal volume $\left(V_{T}\right)$ and pressure support were externally recorded. A pre-set $V_{T}$ of $550 \mathrm{~mL}$ was programmed, with a wide range of pressure support values. RESULTS: All the ventilators showed a deviation of delivered versus programmed $\mathrm{V}_{\mathrm{T}}$ below $10 \%$ in the period without unintentional leaks. In the model with unintentional inspiratory leaks, a progressive drop in delivered $V_{T}$ and pressure support was observed for all ventilators. The reduction in the delivered $V_{T}$ for the highest inspiratory leak ranged between 21 and $40 \%$, corresponding to a decrease in pressure support between 3.09 and $10.15 \mathrm{~cm} \mathrm{H}_{2} \mathrm{O}$ after $5 \mathrm{~min}$. Conversely, in the expiratory model, increases in delivered $\mathbf{V}_{\mathrm{T}}$ and pressure support were observed, ranging between 16 and $33 \%$ and between 2.7 and $6.5 \mathrm{~cm} \mathrm{H}_{2} \mathrm{O}$, respectively. CONCLUSIONS: The introduction of random leaks influences the performance of commercial ventilators with single-limb circuits and intentional leak. The decrease in delivered $\mathbf{V}_{T}$ with inspiratory leaks reaches a magnitude that may have clinically important impacts. Key words: dual-control mode; noninvasive ventilation; leaks; volume-targeted pressure support. [Respir Care 2015;60(2):191-200. () 2015 Daedalus Enterprises]
\end{abstract}

\section{Introduction}

Modern noninvasive ventilators for domiciliary and inhospital use provide the clinician with a wide spectrum of

Drs Luján, Sogo, Grimau, Pomares, and Monsó are affiliated with Servei de Pneumologia, Corporaciò Sanitària Parc Taulí, Hospital de Sabadell, Universitat Autònoma de Barcelona, Parc Taulí, Sabadell; Drs Luján, Blanch, and Monsó are affiliated with Centro de Investigación Biomédica en Red Enfermedades Respiratorias, Instituto de Salud Carlos III, Madrid; Dr Blanch is affiliated with the Critical Care Department, Corporaciò Sanitària Parc Taulí, Hospital de Sabadell, Universitat Autònoma de Barcelona, Parc Taulí, Sabadell, Spain.

Dr Blanch has developed patented inventions related to monitoring ventilator signals. The license for these patents belongs to Corporació Sanitària Parc Taulí, Sabadell, Spain. Dr Blanch also owns 10\% of BetterCare, a research and development subsidiary of Corporació Sanitària Parc Taulí. All other authors have disclosed no conflicts of interest. ventilation modes for the treatment of patients with respiratory failure. Manufacturers have introduced dual-control ventilation modes based on pressure support (volume-targeted pressure support), in which the ventilator increases or decreases the level of support as a function of the targeted tidal volume $\left(\mathrm{V}_{\mathrm{T}}\right)$ set by the clinician.

\footnotetext{
This research was supported by grants from Agència de Gestió d'Ajuts Universitaris i de Recerca (VALOR 2010-00188), Centro de Investigación Biomédica en Red de Enfermedades Respiratorias, and Fundació Parc Taulí.
}

Correspondence: Manel Luján MD PhD, Servei de Pneumologia, Corporació Parc Taulí, Hospital de Sabadell, Parc Taulí 1, 08208 Sabadell, Spain. E-mail: mlujan@tauli.cat.

DOI: $10.4187 /$ respcare. 03413 
In clinical practice, the use of volume-targeted pressure support in noninvasive ventilation (NIV) has produced controversial results. Initial reports demonstrated a benefit mainly in patients with obesity-hypoventilation syndrome, ${ }^{1}$ but later analyses in which pressure support was carefully titrated and monitored did not show this effect. ${ }^{2}$ Other studies focusing on patients with $\mathrm{COPD}^{3-5}$ also demonstrated modest benefits for this dual-control mode.

To simplify the management of ventilators by both clinicians and patients, single-limb circuits with exhalation leak port have been the most frequently used systems for providing a gas mixture from the ventilator to the patient and for preventing rebreathing from the patient's exhaled gas. One of the main drawbacks of this system, however, is that certain essential values (for instance, $V_{T}$ and unintentional leaks) are estimated rather than directly measured. Focusing on this issue, several bench studies have demonstrated that the estimation of $\mathrm{V}_{\mathrm{T}}$ by home ventilators may be modified by the leak. In a model of continuous leaks simulated through a calibrated orifice, 2 studies have shown underestimations of $\mathrm{V}_{\mathrm{T}}$ of different magnitudes by commercial devices, ${ }^{6,7}$ and, in a dynamic leak model, with excess leaks during inspiration or expiration, Sogo and colleagues $^{8}$ found that the increase in inspiratory leaks led to an overestimation of the $\mathrm{V}_{\mathrm{T}}$. Inaccurate $\mathrm{V}_{\mathrm{T}}$ estimations may also manifest in the volume-targeted pressure support modes. In a model of continuous leaks, 2 studies have demonstrated that single-limb circuits with an exhalation leak port provided the targeted $\mathrm{V}_{\mathrm{T}}$, even when continuous additional unintentional leaks were introduced in the circuit. ${ }^{9,10}$ However, Fauroux and colleagues ${ }^{11}$ demonstrated that some ventilators were not able to guarantee the pre-set $\mathrm{V}_{\mathrm{T}}$ when unintentional leaks were introduced, a phenomenon that the authors attributed mainly to the development of asynchronies (autotriggering) rather than to an inaccurate estimation of $\mathrm{V}_{\mathrm{T}}$. The behavior of leaks may be different in clinical practice, however, in which poorly fitted masks may generate leaks only during inspiration, or during exhalation in patients wearing nasal masks who exhale through the mouth. In this setting, we performed a bench study with a model of dynamic leaks (predominant in inspiration or expiration) to determine their influence in volume-targeted pressure support modes.

\section{Methods}

\section{Study Design}

This study was performed in the Hospital de Sabadell, Sabadell, Barcelona, Spain. The experiment was conducted under simulation conditions in a mechanical ventilation laboratory equipped with a breathing simulator (series 1101, Hans Rudolph, Inc., Shawnee, Kansas), which was used to test the ventilators. As a signal acquisition system, an ex-

\section{QUICK LOOK}

\section{Current knowledge}

Leak compensation during noninvasive ventilation (NIV) is essential to assure patient comfort and tolerance. The ability to trigger and cycle the breath in the face of a leak avoids asynchrony and improves NIV success.

\section{What this paper contributes to our knowledge}

The presence of dynamic, unintentional leaks during NIV using a single-limb circuit with a leak port circuit interferes with ventilator performance in the volumetargeted pressure support mode. Inspiratory leaks result in a reduction in pressure support, with no guarantee of delivered tidal volume. A minimal level of pressure support may be required to avoid insufficient support.

ternal polygraph (16Sp Powerlab, ADInstruments, Sydney, Australia), equipped with 2 pressure transducers (1050 model) and 2 pneumotachographs (S300, instrumental dead space $=70 \mathrm{~mL}$, resistance $=0.0018 \mathrm{~cm} \mathrm{H}_{2} \mathrm{O} / \mathrm{L} / \mathrm{s}$ ) was used. Sampling frequency was set to $200 \mathrm{~Hz}$, and the polygraph was connected to a personal computer equipped with Chart 7.0 software for Windows.

The ventilators tested were connected to the simulator through a single standard 2-m tube with a commercial leak port (Swivel, Philips Respironics, Murrysville, Pennsylvania) placed at their distal end. The equation defining the mathematical relationship between pressure and baseline intentional leak for this port was as follows: leak $=-0.034 p^{2}+2.253 p+6.264\left(R^{2}=0.999\right)$. The leak port was maintained during the entire experiment, simulating the intentional leak used in clinical practice in NIV with single-limb configuration. A T-piece was inserted after this leak port to simulate the dynamic unintentional leaks during the inspiratory or expiratory phases.

In the model for inspiratory leaks, a $7.5 \mathrm{~cm} \mathrm{H}_{2} \mathrm{O}$ PEEP threshold valve (Whisperflow, Philips Respironics) was attached to this T-piece. Three levels of leak were added: (1) no leak, with the distal end of the T-piece occluded, maintaining only the port leak (Swivel valve) in the circuit; (2) baseline leak (Swivel valve) + additional leak 1, a calibrated hole with a diameter of $2 \mathrm{~mm}$ at the distal end; and (3) baseline leak (Swivel valve) + additional leak 2, a calibrated hole with a diameter of $3 \mathrm{~mm}$ (Fig. 1).

In the model for expiratory leaks, an active valve (Philips Respironics) was attached to the T-piece, with their opening and closure mechanically controlled by a standard tube connected to the proximal end of the tubing (Fig. 2). 


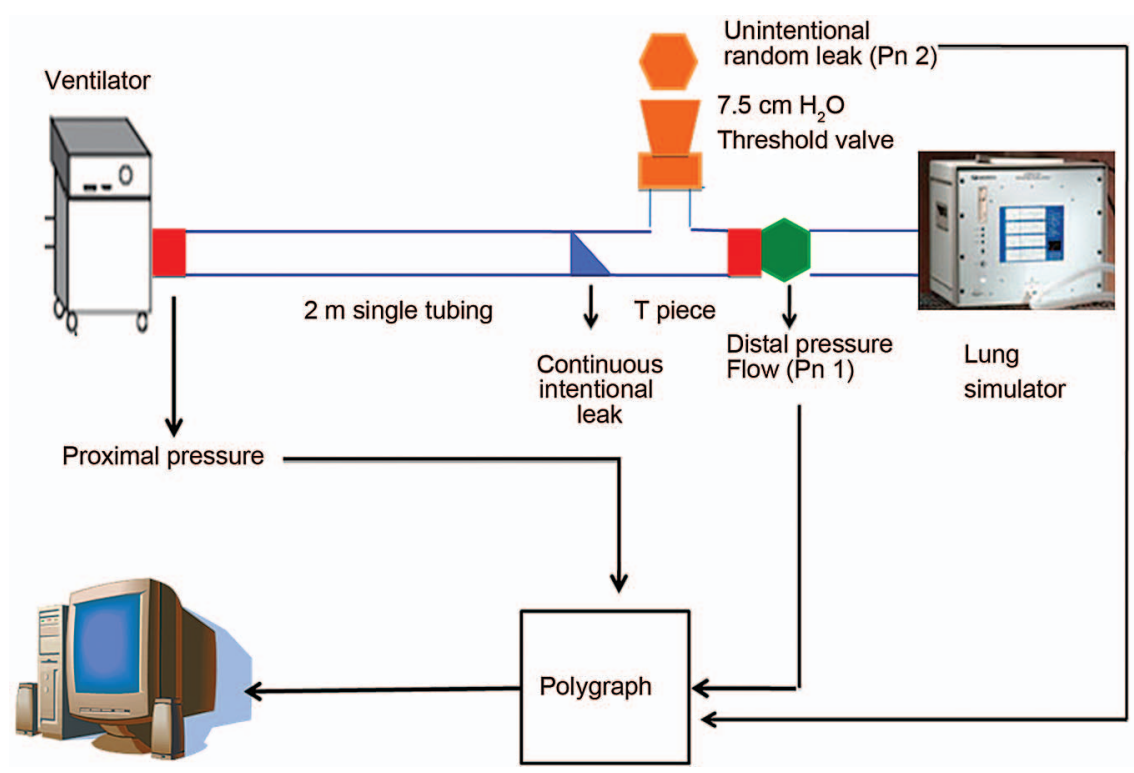

Fig. 1. Design of the experiment in the inspiratory model.

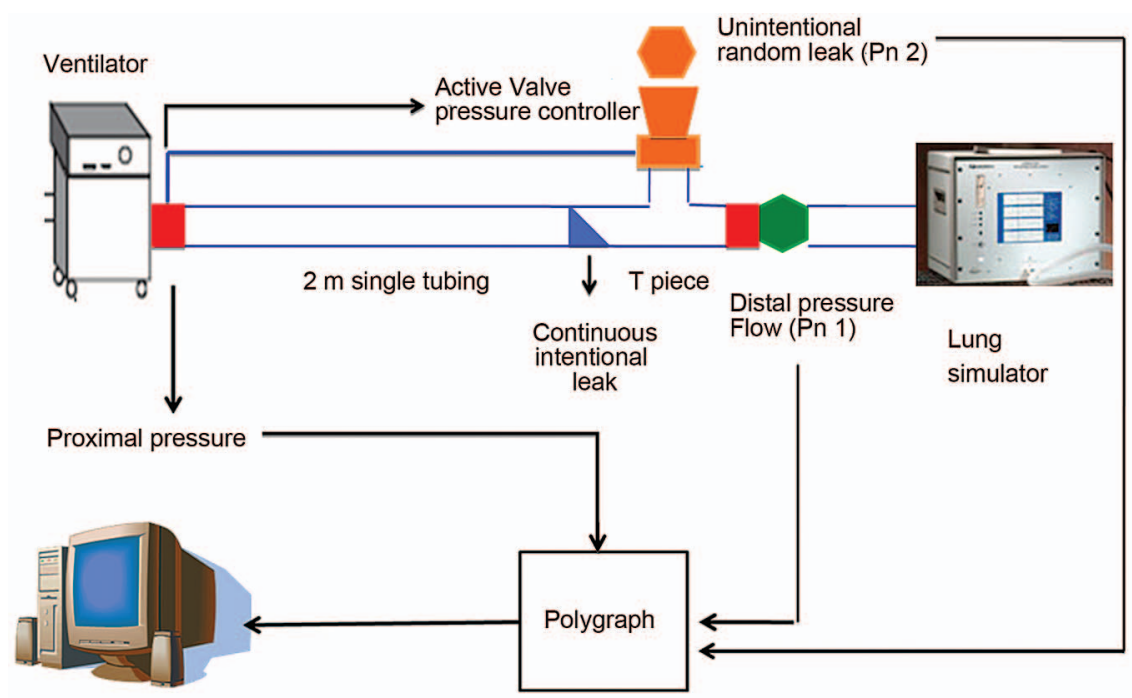

Fig. 2. Design of the experiment in the expiratory model.

In both cases (inspiratory and expiratory leaks), a second pneumotachograph monitored the inspiratory unintentional leaks.

Before each session, both pneumotachographs 1 and 2 and the pressure transducers were calibrated following the manufacturer's instructions, using a 3-L syringe and against a water column.

\section{Parameters Used in the Study}

Parameters of the simulator. Spontaneous breathing frequency was programmed at 15 breaths $/ \mathrm{min}$ in the simulator $\left(-6 \mathrm{~cm} \mathrm{H}_{2} \mathrm{O}\right.$ of effort level/20\% of the respiratory cycle). As volume-targeted pressure support is most frequently used in restrictive patients, compliance was set at $25 \mathrm{~mL} / \mathrm{cm} \mathrm{H}_{2} \mathrm{O}$, and resistance at $7.5 \mathrm{~cm} \mathrm{H}_{2} \mathrm{O} / \mathrm{L} / \mathrm{s} .{ }^{12}$

Parameters of the ventilator. Parameters selected for each ventilator are summarized in Table 1.

\section{Ventilators Tested}

Six commercial ventilators were tested: (1) Vivo 50 (General Electric, Mölnlycke, Sweden), (2) Stellar 150 (ResMed, North Ryde, Australia), (3) Trilogy 100 (Philips Respironics), (4) Puritan Bennett (PB) 560 (Covidien, 
Dynamic Leaks and Dual-Control Modes

Table 1. Parameters Selected for Each Ventilator for the Bench Study

\begin{tabular}{|c|c|c|c|c|c|c|c|c|}
\hline Ventilator & $\begin{array}{l}\text { Targeted } \\
\text { Volume* }\end{array}$ & $\begin{array}{c}\text { Minimum } \\
\text { IPAP } \\
\left(\mathrm{cm} \mathrm{H}_{2} \mathrm{O}\right)\end{array}$ & $\begin{array}{l}\text { Maximum } \\
\text { IPAP } \\
\left(\mathrm{cm} \mathrm{H}_{2} \mathrm{O}\right)\end{array}$ & $\begin{array}{l}\text { EPAP (PEEP) } \\
\quad\left(\mathrm{cm} \mathrm{H}_{2} \mathrm{O}\right)\end{array}$ & $\begin{array}{l}\text { Rise } \\
\text { Time }\end{array}$ & $\begin{array}{c}\text { Inspiratory } \\
\text { Trigger } \dagger\end{array}$ & $\begin{array}{c}\text { Cycle-off } \\
\text { Criterion } \\
(\%)\end{array}$ & $\begin{array}{c}\text { Maximum } \\
\text { Inspiratory } \\
\text { Time (s) }\end{array}$ \\
\hline Vivo 50 & 550 & 8 & 40 & 5 & $2+$ & $9 \ddagger$ & $7 \ddagger(30)$ & 1.5 \\
\hline Stellar 150 & 6.5 & 8 & 40 & 5 & $150 \S$ & Very low & Low & 1.5 \\
\hline Trilogy 100 & 550 & 8 & 34 & 5 & $2 \hbar$ & 9\| & 30 & NA \\
\hline PB 560 & 550 & 8 & 40 & 5 & $1 末$ & $5 \ddagger$ & 30 & 1.5 \\
\hline Carina & 550 & NA & NA & 5 & $200 \S$ & Normal & NA & 1.5 \\
\hline V60 & 550 & 8 & 40 & 5 & $2 \ddagger$ & NA & NA & NA \\
\hline
\end{tabular}

Backup breathing frequency was programmed at 12 breaths/min in the ventilators.

* Measurements are given in $\mathrm{mL}$ for targeted volume, except for Stellar 150, which is given in $\mathrm{L} / \mathrm{min}$ (minute ventilation).

$\dagger$ Inspiratory trigger was set at the lowest sensitivity to avoid autotriggering.

† In analogic scale.

$\S$ Measurements are given in ms.

$\|$ Measurement is given in $\mathrm{L} / \mathrm{min}$.

$\mathrm{IPAP}=$ inspiratory positive airway pressure

$\mathrm{EPAP}=$ expiratory positive airway pressure

$\mathrm{PB}=$ Puritan Bennett

$\mathrm{NA}=$ not available

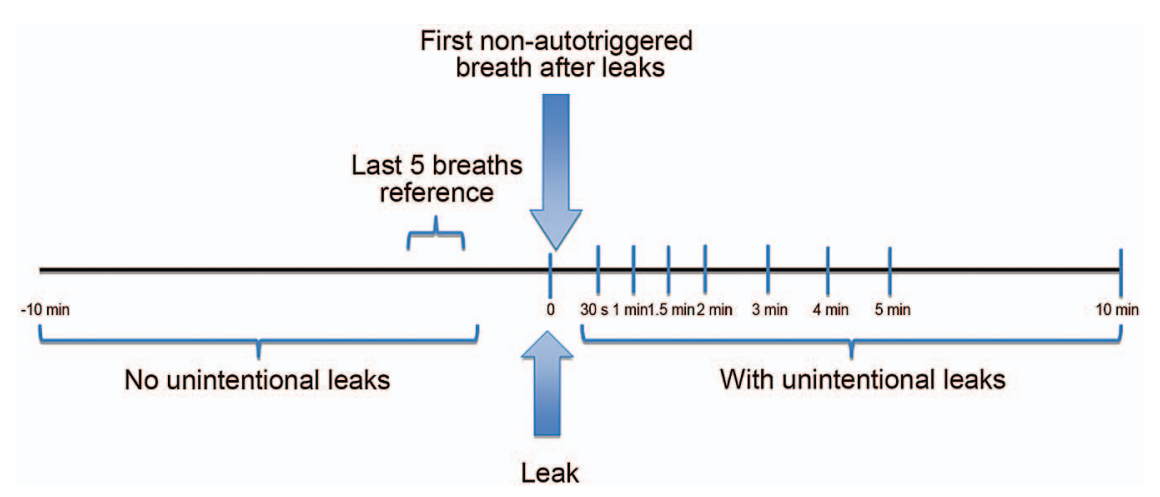

Fig. 3. Measurement protocol.

Mansfield, Massachusetts), (5) Carina (Dräger, Lübeck, Germany, and (6) V60 (Philips Respironics).

\section{Measurement Protocol}

To reach stability with targeted volume and only the leak port circuit, an initial period of $10 \mathrm{~min}$ was recorded. After that, a 10-min period was scheduled for each tested condition (lower and higher inspiratory leak, and expiratory leak). The 4 last breaths of the initial $10-\mathrm{min}$ period with only the leak port circuit (stability period), the first non-autotriggered breath after valve opening (to ensure that the results were not influenced by a lack of compensation of unintentional leaks and labeled as "after leaks"), and the 5 last breaths of each 30-s period (until minute 2) and 1-min period (until minute 5) were recorded. If the variation in $\mathrm{V}_{\mathrm{T}}$ or pressure support was $<5 \%$ after min 5 , only values until this minute were analyzed. The measurement protocol is summarized in Figure 3.
To standardize the analysis of true $\mathrm{V}_{\mathrm{T}}$ entering the respiratory system, the $\mathrm{V}_{\mathrm{T}}$ obtained by the pneumotachograph was converted to BTPS (body-temperature-andpressure-saturated) conditions ${ }^{13}$ as detailed elsewhere. ${ }^{7}$ At the same time, dynamic (unintentional) leak values were monitored (pneumotachograph 2) and registered.

\section{Statistical Analysis}

Statistical analyses were performed using SPSS 19 (SPSS, Chicago, Illinois). Quantitative values for each experimental condition were expressed as means \pm SD. To compare the behavior of the ventilators at different steps of the protocol, the general model for repeated measures was used. When the sphericity of the model could not be assumed (Mauchly's test with $P$ value $<.05$ ), the significance of the model was assessed with the GreenhouseGeisser correction. Inter-subject differences between ven- 


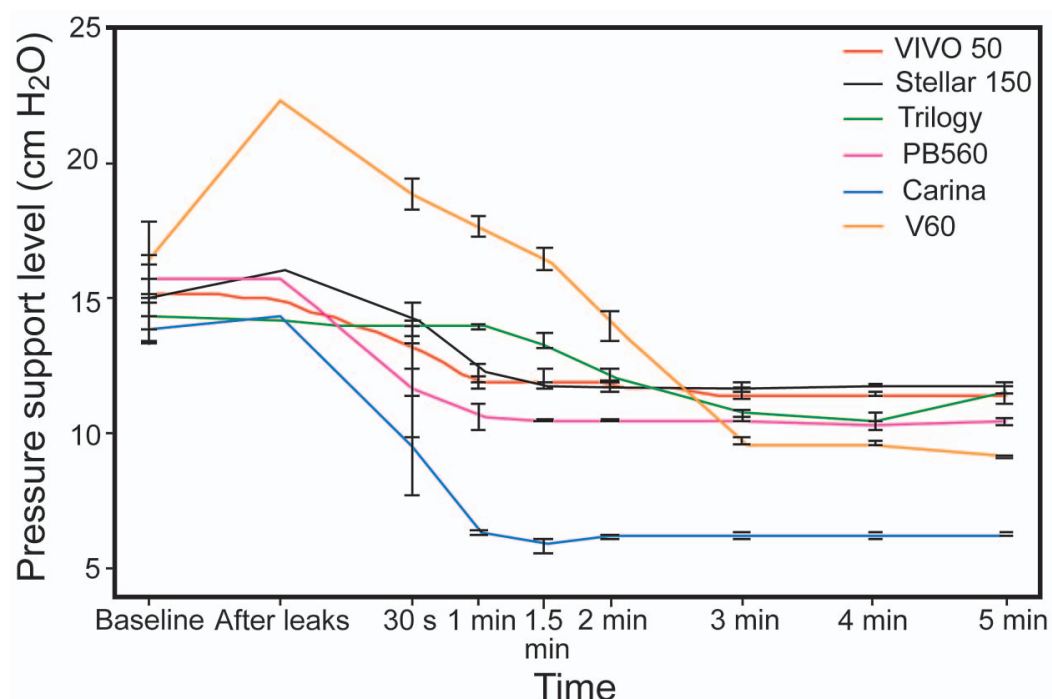

Fig. 4. Changes in the pressure support level after the introduction of unintentional leaks (lower inspiratory leak). Significant intra- and inter-subject differences were found for all ventilators $(P<.01)$. Data are shown as mean \pm SD.

Table 2. Mean Level of Leaks (Measured [Unintentional] and Estimated [Intentional]) at $30 \mathrm{~s}$ After the Valve Opening

\begin{tabular}{|c|c|c|c|c|c|}
\hline \multirow{2}{*}{ Ventilator } & \multirow{2}{*}{$\begin{array}{c}\text { Excess of Inspiratory Leaks } \\
\text { (Lower Leak)* } \\
(\mathrm{L} / \mathrm{min})\end{array}$} & \multirow{2}{*}{$\begin{array}{c}\text { Excess of Inspiratory Leaks } \\
\text { (Higher Leak)* } \\
(\mathrm{L} / \mathrm{min})\end{array}$} & \multirow{2}{*}{$\begin{array}{l}\text { Excess of Expiratory Leaks } \\
\qquad(\mathrm{L} / \mathrm{min})\end{array}$} & \multicolumn{2}{|c|}{$\begin{array}{l}\text { Estimated Intentional } \\
\text { Baseline Leaks } \dagger \\
\quad(\mathrm{L} / \mathrm{min})\end{array}$} \\
\hline & & & & $\begin{array}{c}\text { Inspiratory } \\
\text { (peak) }\end{array}$ & $\begin{array}{c}\text { Expiratory } \\
\text { (mean) }\end{array}$ \\
\hline Vivo 50 & $20.76 \pm 1.72$ & $30.63 \pm 3.17$ & $10.12 \pm 0.06$ & 37.72 & 16.2 \\
\hline Stellar 150 & $24.72 \pm 0.88$ & $32.86 \pm 1.88$ & $13.41 \pm 0.57$ & 37.90 & 17.05 \\
\hline Trilogy 100 & $21.66 \pm 0.24$ & $31.90 \pm 1.01$ & $14.96 \pm 0.83$ & 37.22 & 17.1 \\
\hline PB 560 & $21.48 \pm 2.95$ & $28.30 \pm 2.7$ & $13.09 \pm 0.09$ & 38.37 & 16.8 \\
\hline Carina & $20.20 \pm 2.06$ & $25.40 \pm 3.85$ & $11.05 \pm 0.05$ & 36.89 & 17.2 \\
\hline V60 & $27.67 \pm 1.45$ & $41.50 \pm 1.14$ & $14.80 \pm 0.16$ & 38.81 & 16.6 \\
\hline \multicolumn{6}{|c|}{$\begin{array}{l}\text { Lower and higher leaks were simulated trough calibrated holes ( } 2 \text { and } 3 \mathrm{~mm} \text {, respectively), placed after the threshold valve. } \\
\text { Estimated in the period without unintentional leaks. } \\
\text { PB = Puritan Bennett }\end{array}$} \\
\hline
\end{tabular}

tilators were assessed by Bonferroni post hoc analysis. Statistical significance was set at a $P$ value of $<.05$.

\section{Results}

The protocol was completed for all tested ventilators, which reached stability in pressure support and predetermined $\mathrm{V}_{\mathrm{T}}$ values at the end of 10 min with the leak port circuit, and before minute 5 in the periods with dynamic unintentional leaks. Differences in targeted and measured volume at the end of the period with baseline leak (only the exhalation port) were below $10 \%$ for all the ventilators studied, although there were some differences between the pressure support levels reached by each ventilator (Fig. 4).

In the period with dynamic leaks (valve open), the amount of leaks varied according to the level of pressure support attained by the devices. Mean level of leaks (measured [unintentional] and estimated [intentional]) at $30 \mathrm{~s}$ after the valve opening are displayed in Table 2 . As shown in the higher leak, leaks during the inspiratory phase accounted for up to $70 \mathrm{~L} / \mathrm{min}$.

The results of the model with inspiratory leaks are displayed in Figure 4 (for pressure support) and Figure 5 (for $\mathrm{V}_{\mathrm{T}}$ ) for the lower leak, and in Figures 6 and 7 for the higher leak. As can be observed, the addition of inspiratory dynamic leaks caused a progressive decrease both in the pressure support and in the delivered $\mathrm{V}_{\mathrm{T}}$, falling clearly below the programmed targeted values. The progression of the decrease differed between the devices, with some ventilators (Carina and PB 560) reaching stability before minute 2 and others (Trilogy and V60) showing a slower decrease. At the end of the 5-min period, differences in the 


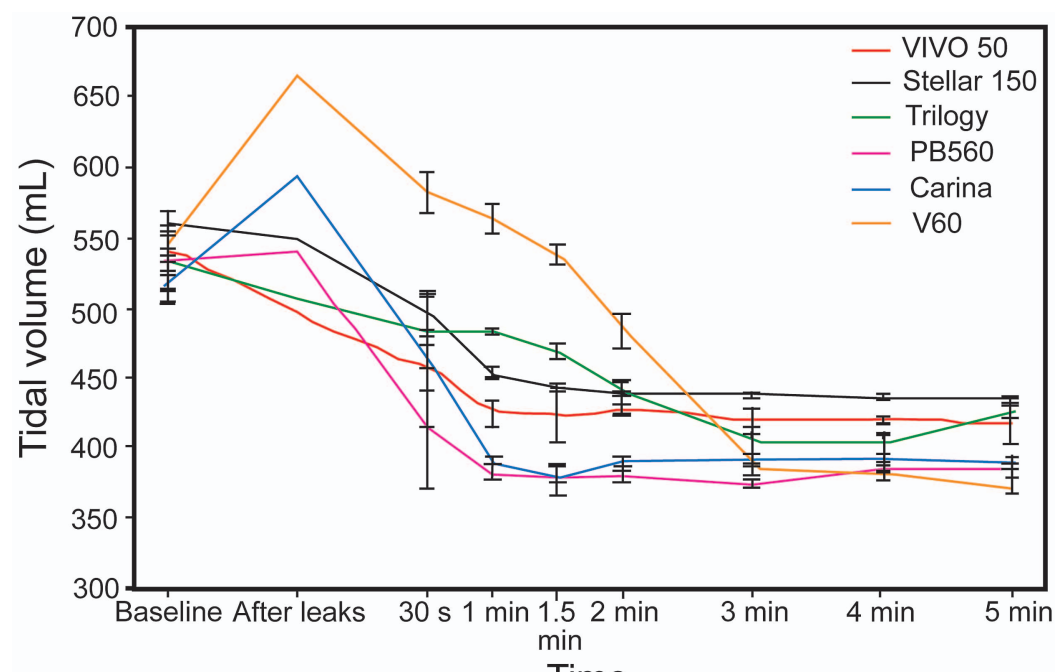

Time

Fig. 5. Changes in the delivered tidal volume after the introduction of unintentional leaks (lower inspiratory leak). Significant intra- and inter-subject differences were found for all ventilators $(P<.01)$. Data are shown as mean \pm SD.

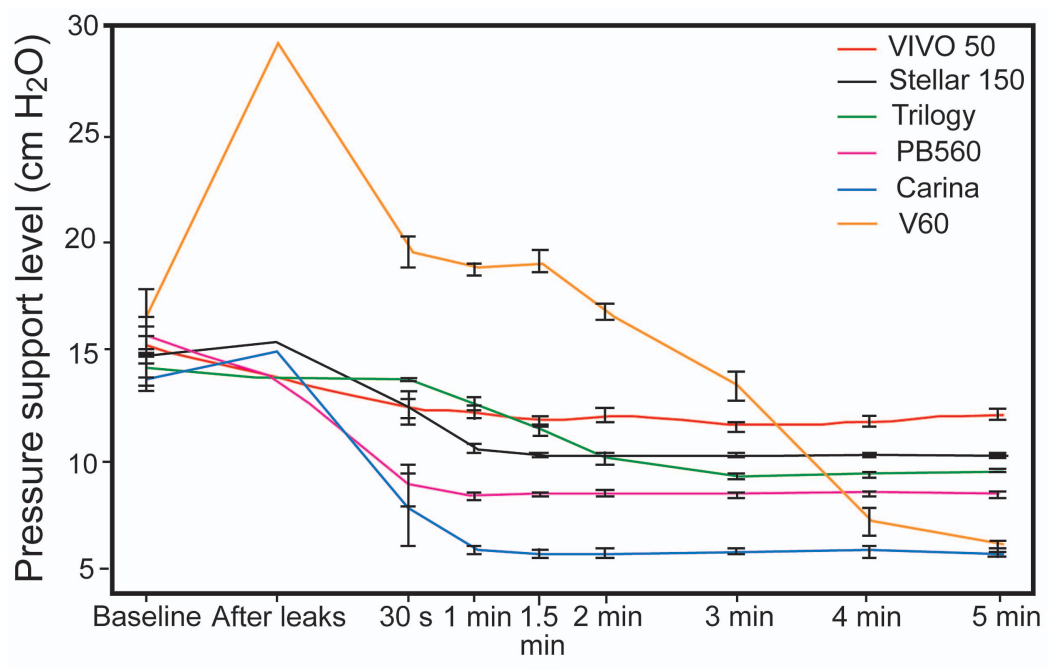

Time

Fig. 6. Changes in the pressure support level after the introduction of unintentional leaks (higher inspiratory leak). Significant intra- and inter-subject differences were found for all ventilators $(P<.01)$. Data are shown as mean \pm SD.

mean delivered $\mathrm{V}_{\mathrm{T}}$ comparing the periods with and without dynamic leaks ranged from 21 to $31 \%$ for lower leak and from 21 to $40 \%$ for higher leak. Regarding the pressure support level, the differences ranged between 2.82 and $7.28 \mathrm{~cm} \mathrm{H}_{2} \mathrm{O}$ for lower leak and between 3.09 and $10.16 \mathrm{~cm} \mathrm{H}_{2} \mathrm{O}$ for higher leak. Interestingly, 2 ventilators (Carina and V60) showed an increase both in pressure support levels and in delivered $\mathrm{V}_{\mathrm{T}}$ immediately after valve opening. The other 4 ventilators showed minimal differences (Stellar 150) or only modest decreases (Vivo 50, Trilogy, and PB 560) in these 2 variables. Statistically significant intra-subject (ventilator) differences were found as a function of time for all tested ventilators $(P<.01$ by
Greenhouse-Geisser test). Similarly, significant differences between the different ventilator models were observed (Bonferroni post hoc test $<.01$ ). The influence of the level of leak in the delivered $\mathrm{V}_{\mathrm{T}}$ by each ventilator is shown in Figure 8. As displayed, all ventilators presented significant differences $(P<.01)$ when the 2 levels of dynamic leaks were compared, with the exception of Vivo 50.

In the model with expiratory leaks, as observed in Table 3 , the addition of the leaks caused an increase in both the delivered $\mathrm{V}_{\mathrm{T}}$ and pressure support. As in the inspiratory model, the progression of the increase differed between the ventilators studied, and, at the end of the 5-min period, the increase in $\mathrm{V}_{\mathrm{T}}$ ranged between 16 and 33\%, compared 


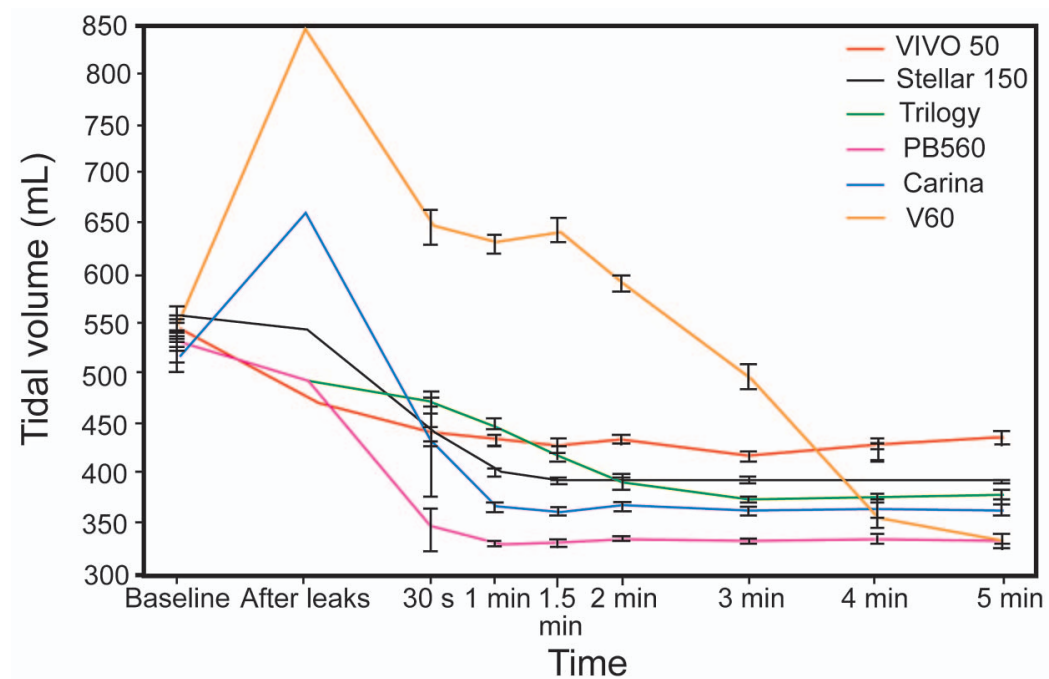

Fig. 7. Changes in the delivered tidal volume after the introduction of unintentional leaks (higher inspiratory leak). Significant intra- and inter-subject differences were found for all ventilators $(P<.01)$. Data are shown as mean \pm SD.

with the volume delivered at the end of the stability period without unintentional leaks. Finally, the increase in pressure support level ranged between 2.7 and $6.5 \mathrm{~cm} \mathrm{H}_{2} \mathrm{O}$. As occurred with the inspiratory model, significant intra-subject differences in $\mathrm{V}_{\mathrm{T}}$ as a function of time were found for all the devices studied $(P<.01$ Greenhouse-Geisser test), and inter-subject differences were found also for all ventilators, with the exception of the comparison between Vivo 50 and Stellar 150 ventilators.

Interestingly, the results of $\mathrm{V}_{\mathrm{T}}$ displayed by the ventilators able to download this information from internal memory (all of them with exception of Carina and V60) showed a transient overestimation when inspiratory leaks were introduced and a underestimation when expiratory leaks were introduced (Fig. 9).

\section{Discussion}

The most important findings of the study are that, in NIV and with single-limb and leak port circuit, the presence of dynamic unintentional leaks altered the delivered $\mathrm{V}_{\mathrm{T}}$ during volume-targeted pressure support mode in both inspiratory and expiratory phases. From the clinical point of view, the most feasible model is the excess inspiratory leak model: in this condition, the delivered $\mathrm{V}_{\mathrm{T}}$ fell by $40 \%$ compared with the values without unintentional leak. This decrease may lead to significant hypoventilation in clinical practice.

The influence of leaks in dual-control modes has been assessed in 2 studies. Carlucci and colleagues ${ }^{9}$ studied the influence of the configuration of bi-level ventilator circuits on the compensation of leaks during volume-targeted ventilation. In their study, when unintentional leaks were in- troduced, the ventilators were able to compensate for them, but only when a vented circuit (leak port circuit) was used. When a non-vented (active expiratory valve) circuit was used, the authors observed a decrease in delivered $\mathrm{V}_{\mathrm{T}}$. Similarly, Khirani and colleagues ${ }^{10}$ studied 3 double-limb circuit ventilators and 2 ventilators with a single circuit and exhalation leak port and demonstrated that these devices were unable to maintain the pre-set minimal $\mathrm{V}_{\mathrm{T}}$ during unintentional leaks; however, the same ventilators studied with a single-limb circuit and leak port were able to do so. Both studies introduced unintentional leaks as a continuous parameter through a calibrated hole of variable diameter. Based on the results of these previous studies, the volume-targeted pressure support mode was suggested to be safe with a single-limb and leak port circuit.

The model with excess leaks during inspiratory or expiratory phases was first explored by Storre et al ${ }^{14}$ in a patient model and generating excess leaks in the circuit with manual occlusion of a T-piece inserted in the circuit. In a similar way, Carteaux et al ${ }^{15}$ designed a model of excess of inspiratory unintentional leaks using a water column of variable height to study the frequency of asynchronies at different leak levels. However, the implications of this kind of leak in volume-targeted pressure support mode have not been addressed.

We hypothesize that the findings in our study are more probably due to monitoring inaccuracies than to a lack of compensation of the unintentional leaks. Even with the highest inspiratory leak, only a modest decrease $(<10 \%)$ in delivered $\mathrm{V}_{\mathrm{T}}$ immediately after the valve's opening was detected in some of the ventilators; in fact, 2 of them, frequently used in clinical practice for the acute setting (Carina and V60), showed a transient overshoot phenom- 

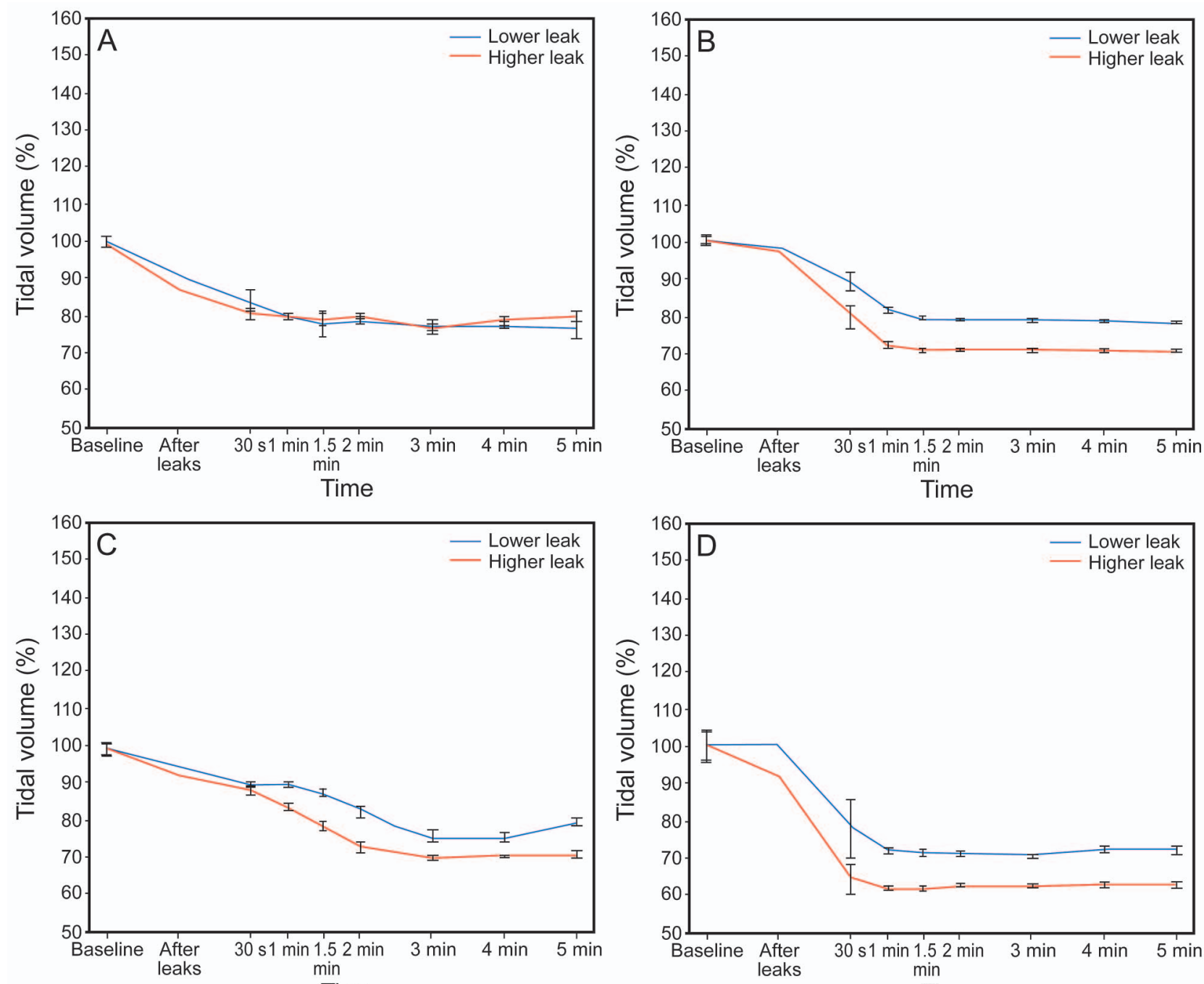

Time
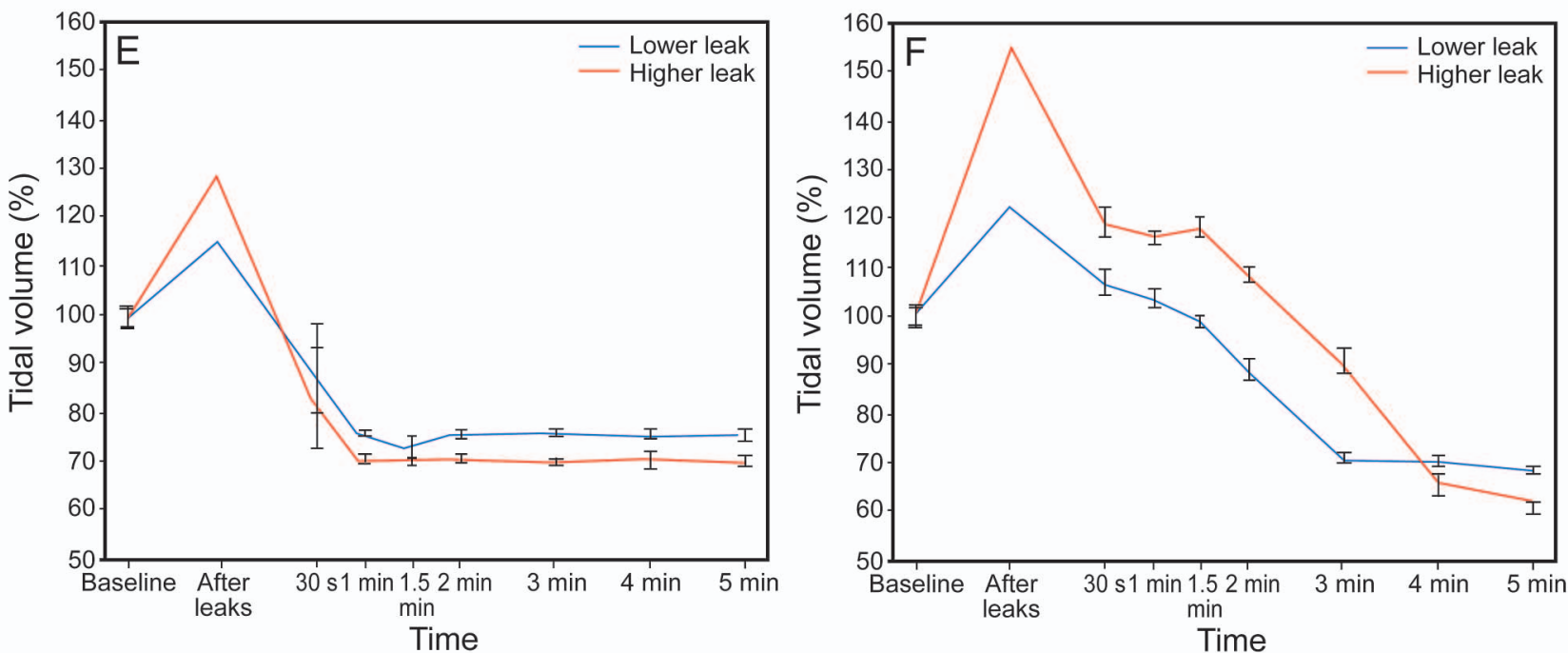

Fig. 8. Comparison of delivered tidal volume (\% from baseline) between lower and higher inspiratory leak for all ventilators. $P<.01$ for all ventilators except the Vivo 50. A: Vivo 50, B: Stellar 150, C: Trilogy 100, D: Puritan Bennett 560, E: Carina, and F: V60. Data are shown as mean \pm SD. 
Dynamic Leaks and Dual-Control Modes

Table 3. Changes in the Delivered Tidal Volume and Pressure Support After the Introduction of Unintentional Leaks (Expiratory Leak)

\begin{tabular}{|c|c|c|c|c|c|c|c|c|c|c|c|c|}
\hline \multirow{2}{*}{ Stage } & \multicolumn{2}{|c|}{ Vivo 50} & \multicolumn{2}{|c|}{ Stellar 150} & \multicolumn{2}{|c|}{ Trilogy } & \multicolumn{2}{|c|}{ PB 560} & \multicolumn{2}{|c|}{ Carina } & \multicolumn{2}{|c|}{ V60 } \\
\hline & Mean $V_{T}$ & Mean PS & Mean $V_{T}$ & Mean PS & Mean $\mathrm{V}_{\mathrm{T}}$ & Mean PS & Mean $\mathrm{V}_{\mathrm{T}}$ & Mean PS & Mean $V_{T}$ & Mean PS & Mean $V_{T}$ & Mean PS \\
\hline No leak & 545 & 15.2 & 561 & 15.0 & 533 & 14.2 & 536 & 15.7 & 515 & 13.8 & 544 & 16.3 \\
\hline After leaks & 550 & 14.4 & 500 & 12.1 & 495 & 10.6 & 514 & 12.5 & 513 & 14.7 & 525 & 13.9 \\
\hline $30 \mathrm{~s}$ & 608 & 16.9 & 568 & 14.5 & 504 & 11.6 & 705 & 21.4 & 633 & 18.2 & 536 & 13.9 \\
\hline $1 \mathrm{~min}$ & 640 & 18.5 & 637 & 17.0 & 526 & 12.8 & 719 & 21.8 & 634 & 18.1 & 532 & 14.1 \\
\hline $1.5 \mathrm{~min}$ & 660 & 19.4 & 651 & 17.6 & 560 & 14.5 & 721 & 21.8 & 626 & 17.8 & 542 & 15.5 \\
\hline $2 \min$ & 670 & 19.6 & 653 & 17.6 & 597 & 15.8 & 723 & 22.0 & 629 & 18.0 & 597 & 18.0 \\
\hline $3 \mathrm{~min}$ & 669 & 19.6 & 656 & 17.7 & 660 & 18.6 & 721 & 21.9 & 632 & 17.9 & 704 & 22.2 \\
\hline $4 \min$ & 669 & 19.6 & 654 & 17.6 & 704 & 20.6 & 718 & 21.9 & 647 & 18.5 & 722 & 23.0 \\
\hline $5 \min$ & 668 & 19.6 & 654 & 17.7 & 705 & 20.8 & 716 & 22.0 & 670 & 19.3 & 694 & 22.1 \\
\hline $\begin{array}{l}\text { Significant intra- } \\
\mathrm{PB}=\text { Puritan } \mathrm{Be} \\
\mathrm{V}_{\mathrm{T}}=\text { tidal volur } \\
\mathrm{PS}=\text { pressure }\end{array}$ & $\begin{array}{l}\text { and inter-subj } \\
\text { nnett } \\
\text { ne } \\
\text { ipport }\end{array}$ & ct differences & ere found for & ill ventilators & & & & & & & & \\
\hline
\end{tabular}

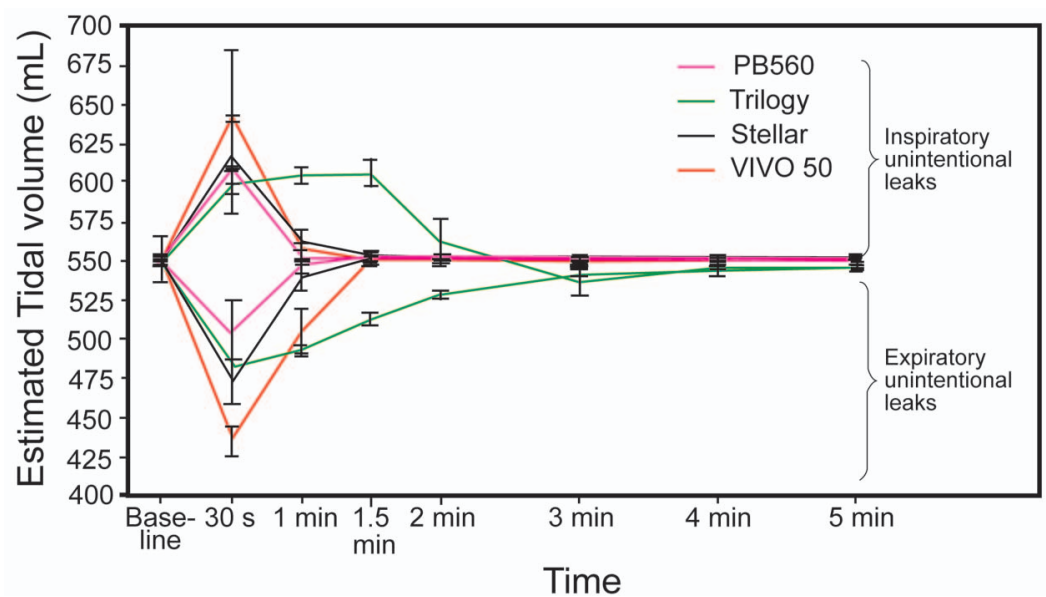

Fig. 9. Estimated tidal volume the ventilators displayed, downloaded from internal memory.

enon with increases both in the pressure support and in the delivered $\mathrm{V}_{\mathrm{T}}$ after the sudden introduction of unintentional leaks. This overshoot has been described by other authors, ${ }^{11}$ and, although it suggests that the level of compensation of the leaks was adequate, it might be uncomfortable for some patients. ${ }^{16}$

In a previous study by our group, ${ }^{8}$ introduction of unintentional dynamic leaks only during one phase of the respiratory cycle affected the accuracy of the estimation of leak and $\mathrm{V}_{\mathrm{T}}$ values. Those findings seem to suggest that the estimation of leak and $\mathrm{V}_{\mathrm{T}}$ by built-in software, or by the ventilators themselves, is based on the assumption that there is a proportional relationship between leaks during expiration and inspiration; in clinical practice, however, it may be that leaks occur only in one phase of the respiratory cycle, mostly during inspiration. The algorithms based on these assumptions overestimated delivered $\mathrm{V}_{\mathrm{T}}$ in the unintentional leak setting, and underestimated it in the case of expiratory leaks. These previous findings reinforce the hypothesis that the changes observed in delivered $V_{T}$ after introducing unintentional leaks are probably a consequence of a deviation in its estimation. ${ }^{17}$

Another interesting result derived from the present study was the different period of time elapsed by the ventilators to reach stability after the introduction of dynamic leaks. In some ventilators, the stability is reached after a few breaths, whereas, in others, it was attained later. This observation might be explained by the different algorithms of increases and decreases in pressure in response to the changes in estimated $\mathrm{V}_{\mathrm{T}}$. Whereas in some ventilators (eg, Carina, Stellar 150, or PB 560) the changes in pressure support to ensure the targeted volume are achieved in few breaths, in others (V60, Trilogy, and even Vivo 50), these changes are more progressive (maximum changes between 1 and $2 \mathrm{~cm} \mathrm{H}_{2} \mathrm{O}$ of pressure support/min) for improving the patient's tolerance. The initial overshoot observed in 


\section{Dynamic Leaks and Dual-Control Modes}

some ventilators after the introduction of unintentional inspiratory leaks may be also related to the features of internal algorithms in each model.

The findings of the present study may have important clinical implications. First of all, if the clinician chooses a volume-targeted pressure support mode, it seems reasonable to set a minimum pressure support level, corresponding to a minimum safe volume. Our data confirm that, in the presence of large leaks and a wide range of pressures, delivered $\mathrm{V}_{\mathrm{T}}$ may fall by up to $40 \%$ in $5 \mathrm{~min}$, placing the patient at risk of severe hypoventilation. Second, the influence of leaks has not been addressed in clinical studies of the efficacy of dual-control modes, and the potential benefits of these hybrid modes have not been consistently demonstrated in the literature. ${ }^{18}$

Some limitations of the study should be pointed out. Even after introducing unintentional dynamic leaks during an entire phase of the cycle, the design may not reflect the wide spectrum of possibilities of patient-interface interactions, because leaks may appear only during small periods of time in each cycle (only at end inspiration, or during the second half of the inspiratory cycle, for example). Similarly, patient-ventilator interactions may be much more complex than the present model allows for, with changes in respiratory drive, compliance, and ventilatory pattern. Finally, the evaluation was performed in a restrictive model, and it may be questionable whether these findings are of application in an obstructive model.

\section{Conclusions}

In NIV using a single-limb with leak port circuit, the presence of dynamic unintentional leaks may interfere with the performance of the ventilators in volume-targeted pressure support modes. The observation that inspiratory leaks may reduce pressure support values, with no guarantee that the pre-set values of $\mathrm{V}_{\mathrm{T}}$ are delivered, may have clinical relevance. In this situation, it seems reasonable to recommend a minimum pressure support level corresponding to a minimum safe $\mathrm{V}_{\mathrm{T}}$.

\section{ACKNOWLEDGMENT}

We are grateful to Michael Maudsley for revision of the English manuscript.

\section{REFERENCES}

1. Storre JH, Seuthe B, Fiechter R, Milioglou S, Dreher M, Sorichter S, Windisch W. Average volume-assured pressure support in obesity hypoventilation: a randomized crossover trial. Chest 2006;130:815 821.

2. Murphy PB, Davidson C, Hind MD Simonds A, Williams AJ, Hopkinson NS, et al. Volume-targeted versus pressure support non-invasive ventilation in patients with super obesity and chronic respiratory failure: a randomised controlled trial. Thorax 2012;67:727734.

3. Ambrogio C, Lowman X, Kuo M, Malo J, Prasad AR, Parthasarathy S. Sleep and non-invasive ventilation in patients with chronic respiratory insufficiency. Intensive Care Med 2009;35:306-313.

4. Crisafulli E, Manni G, Kidonias M, Trianni L, Clini EM. Subjective sleep quality during average volume assured pressure support (AVAPS) ventilation in patients with hypercapnic COPD: a physiological pilot study. Lung 2009;187:299-305.

5. Oscroft NS Ali M, Gulati A, Davies MG, Quinnell TG, Shneerson JM, Smith IE. A randomised crossover trial comparing volume assured and pressure preset noninvasive ventilation in stable hypercapnic COPD. COPD 2010;7:398-403.

6. Contal O, Vignaux L, Combescure C, Pepin JL, Jolliet P, Janssens JP. Monitoring of non-invasive ventilation by built-in software of home bi-level ventilators: a bench study. Chest 2012;141:469-476.

7. Luján M, Sogo A, Pomares X, Monsó E, Sales B, Blanch Ll. Effect of leak and breathing pattern on the accuracy of tidal volume estimation by commercial home ventilators: a bench study. Respir Care 2013;58:770-777

8. Sogo A, Montanya J, Monsó E, Blanch Ll, Pomares X, Lujan M. Effect of dynamic random leaks on the monitoring accuracy of home mechanical ventilators: a bench study. BMC Pulm Med 2013;13:75.

9. Carlucci A, Schreiber A, Mattei A Malovini A, Bellinati J, Ceriana $\mathrm{P}$, Gregoretti C. The configuration of bi-level ventilator circuits may affect compensation for non-intentional leaks during volume-targeted ventilation. Intensive Care Med 2013;39:59-65.

10. Khirani S Louis B, Leroux K, Delord V, Fauroux B, Lofaso F. Harms of unintentional leaks during volume targeted pressure support ventilation. Respir Med 2013;107:1021-1029.

11. Fauroux B, Leroux K, Pépin JL, Lofaso F, Louis B. Are home ventilators able to guarantee a minimal tidal volume? Intensive Care Med 2010;36:1008-1014.

12. Oliveri C, Costa R, Conti G, Navalesi P. Bench studies evaluating devices for non-invasive ventilation: critical analysis and future perspectives. Intensive Care Med 2012;38:160-167.

13. Brown SP, Miller WC, Eason JM. Exercise physiology: basis of human movement in health and disease. Baltimore: Lippincott Williams \& Wilkins; 2006:98-126.

14. Storre JH, Bohm P, Dreher M, Windisch W. Clinical impact of leak compensation during non-invasive ventilation. Respir Med 2009; 103:1477-1483

15. Carteaux G, Lyazidi A, Cordoba-Izquierdo A, Vignaux L, Jolliet P, Thille AW, et al. Patient-ventilator asynchrony during noninvasive ventilation: a bench and clinical study. Chest 2012;142:367-376.

16. Janssens JP, Metzger M, Sforza E. Impact of volume targeting on efficacy of bi-level non-invasive ventilation and sleep in obesityhypoventilation. Respir Med 2009;103:165-172.

17. Lujan M, Pomares X. Noninvasive mechanical ventilation: reflections on home monitoring. Arch Bronconeumol 2014;50:85-86

18. Windisch W, Storre JH. Target volume settings for home mechanical ventilation: great progress or just a gadget? Thorax 2012;67:663665 